\title{
Physical Modeling of Laser-Induced Breakdown of Glass
}

\author{
Jaemyoung Lee ${ }^{1, \star}$, Michael F. Becker ${ }^{2}$, and Taikyeong T. Jeong ${ }^{2}$ \\ ${ }^{1}$ Korea Polytechnic University, \\ 2121 Jungwang Shihung Kyuggi, Korea(ROK) 429-793 \\ lee@kpu.ac.kr \\ ${ }^{2}$ Department of Electrical and Computer Engineering \\ University of Texas at Austin
}

\begin{abstract}
We made a physical model for investigation of laser-induced breakdown of glass. To estimate the laser energy absorption through electron heating, we derive a power transfer rate equation and an electron number density equation for a steady state as a function of temperature and electric field. Numerical simulations using the derived equations show that the laser power absorption dependence of glass on temperature and electric field strength.
\end{abstract}

\section{Introduction}

Laser ablation has emerged as one of promising techniques for material processing such as thin film depositions, nanoparticle fabrications, etc. Laser ablation has proven its advantages in material processing because it does not contaminates materials during the process. The foundations of laser ablation, however, lie in the old field of laser-material interactions where many materials were irradiated with high power laser pulses. Therefore, studies about the laser-material interactions have been required to investigate the laser-material interactions.

In this paper, we form a physical model to analyze the laser-induced breakdown of glass, provide simple equations for numerical analysis using experimental results published from other groups, and show simulation results about energy absorption, electron number density in terms of temperature and electric field using the derived equations.

\section{Theoretical Background}

The interaction between the laser field and free electrons can explain the high power laser breakdown mechanism. Electron avalanche theory [1,2, 3] has been accepted to explain the high power laser breakdown of transparent(wide band gap) solids at both visible and near-infrared wavelengths. This theory assumes that breakdown starts above the critical laser intensity where the energy gain

\footnotetext{
^ Corresponding author.
} 
rate from the laser field by a few electrons exceeds the energy loss rate due to lattice phonon scattering. These starting electrons with density $n_{0}$ increase their kinetic energies in the high electric field, and initiate impact ionization leading to an exponential increase of the free electron density.

S.C. Jones et al. 3] found discrepancies in this theory for explaining laser induced breakdown of wide band gap materials such as ultrapure alkali halides and $\mathrm{SiO}_{2}$ at visible and near-infrared wavelengths. In one experiment, they measured a lattice temperature that increased gradually as the laser intensity increased and found that breakdown occurred around the melting temperature. They observed that the lattice temperature gradually increased with increasing laser intensity. They explained this phenomenon as a multiphoton absorption process rather than impact ionization process.

Lattice heating was caused by simultaneous free-electron mediated energy transfer from the laser field to the lattice, free-electron heating [4,5]. B. Gorshkov, et al. [5], investigated carrier generation processes, both impact ionization and multiphoton absorption, for laser-induced breakdown and concluded that the breakdown mechanism begins to change from an impact ionization process to a multiphoton generation process at near infrared wavelengths such that, at visible wavelengths, the multiphoton absorption mechanism is dominant in wide band gap materials.

Pulse width is also an important parameter because electron density increases exponentially with time for impact ionization while linearly for multiphoton absorption. At wavelengths longer than $2 \mu \mathrm{m}$, the electron multiplication rate and energy transfer rate approach the dc limit, since as the laser frequency decreases, the momentum relaxation rate becomes larger than the laser frequency. In this region, the photon field of the laser is assumed to be a sinusoidal electric field in which the electrons move. This implies that photon energy is small compared to the electron kinetic energy such that energy change from the field can be continuous in time. At short wavelengths $(<500 \mathrm{~nm})$, impact ionization and freeelectron heating are less dominant because the impact ionization threshold moves to higher electric fields, making multiphoton absorption the dominant mechanism. At these wavelengths, even though the multiphoton absorption mechanism is dominant, free electron heating is another efficient mechanism to contribute to lattice heating and melting at laser intensities below the impact ionization threshold.

\section{Physical Model for Numerical Analysis}

In the case of glass, D. Arnold et al. [6], showed that neither the quantummechanical approach nor the standard classical approach followed their exact derivation of the power transfer rate from the laser field to electrons at a wavelength of $1 \mu \mathrm{m}$. The power transfer rate at this wavelength lay between these two approaches.

In our numerical simulation of irradiation with a $1 \mu \mathrm{m}$ laser, the exact power transfer rate of D. Arnold and E. Cartier [6] was used. This approach eliminated 


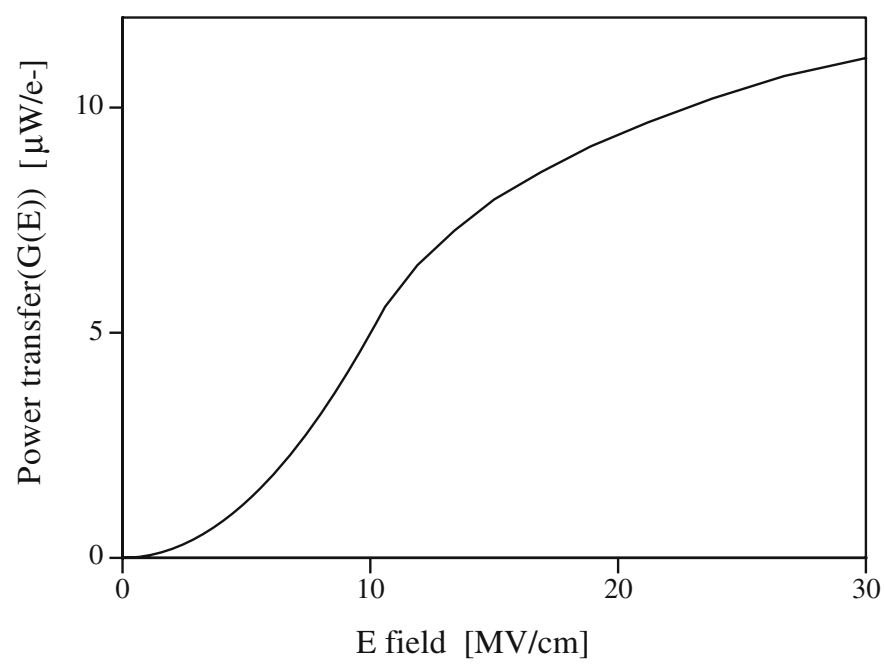

Fig. 1. Power transfer per electron versus E field

additional complex calculations, such as the laser field to free electron interaction equation, to get the power transfer rate. Equation 1 was devised to imitate their result for energy transfer from electrons to the lattice. In Figure 1, the equation 1 is plotted.

$$
\begin{aligned}
G(E) & =5 \times 10^{-14} \times E^{2}, \quad \text { if } E<10^{7}[\mathrm{~V} / \mathrm{cm}] \\
& =3 \times \ln \left(E+210^{6}\right)-39.7, \quad \text { if } E>10^{7}[\mathrm{~V} / \mathrm{cm}]
\end{aligned}
$$

The evolution of the free-electron density, $n_{e}(t)$, is essential to modeling a microsphere ablated by a high power laser pulse. Generation and recombination processes are included in the ionization rate equation as shown below,

$$
\frac{\partial n}{\partial t}=P_{g e n}-P_{l o s s}+P_{t h e r m}
$$

where $P_{\text {gen }}$ is laser induced ionization generation rate, $P_{\text {loss }}$ is loss rate due to electron hole recombination, and $P_{\text {therm }}$ is thermal ionization rate. These equations can be described by

$$
\begin{array}{r}
P_{\text {gen }}=\gamma(E) \times n_{e} \\
P_{\text {loss }}=\sigma(E) \times v_{e} \times n_{e} \times n_{h} \\
P_{\text {therm }}=N_{c}(T) \exp \left(-\frac{E_{g}}{2 k T}\right)
\end{array}
$$

where $\gamma(E)$ is the electric field dependent ionization coefficient, $E$ is electric field, $\sigma(E)$ is the Coulombic capture cross section with field dependence. The quantity 
$v_{e}$ is the effective velocity. In the dc case at low electric fields, $v_{e}$ approaches the drift velocity. In the simulation, the hole density, $n_{h}$, is assumed to be the same as the electron density, $n_{e} . N_{c}(T)$ is density of states and $E_{g}$ is the band gap energy of the material.

D. Buchanan, et al. 7] compared experimental capture cross sections with numerical simulations using a classical Monte Carlo calculation and a quantum Monte Carlo calculation. The capture cross section decreases from $10^{-12}$ to $3 \times 10^{-15} \mathrm{~cm}^{2}$ as the electric fields goes from $2 \times 10^{5}$ to $3 \times 10^{6} \mathrm{~V} / \mathrm{cm}$. Above the threshold electric field $\left(\approx 1.2 \times 10^{6} \mathrm{~V} / \mathrm{cm}\right)$, the field dependence of the capture cross section is a power law with an exponent of -1.5 , and below this threshold, it has an exponent of -3.0 . The ionization rate, $\gamma(E)$, is expressed as,

$$
\gamma=\alpha(E) V_{\text {drift }}
$$

where $V_{\text {drift }}$ is the drift velocity of electrons. The ionization coefficient, $\alpha(E)$, is given by D. Du [8] as

$$
\alpha(E)=\frac{e E}{U_{i}} \exp \left(-\frac{E_{i}}{E\left(1+E / E_{p}\right)+E_{k T}}\right)
$$

The constants $E_{k T}, E_{p}$, and $E_{i}$ are threshold electric fields for electrons to overcome the decelerating effects of thermal, phonon, and ionization scattering, respectively. For the laser fluences used in this simulation, the ionization rate, equation 2. reaches steady state in much less than $1 \mathrm{~ns}$. Therefore, it is solved for a steady state carrier density at each time step of the simulation. Solving

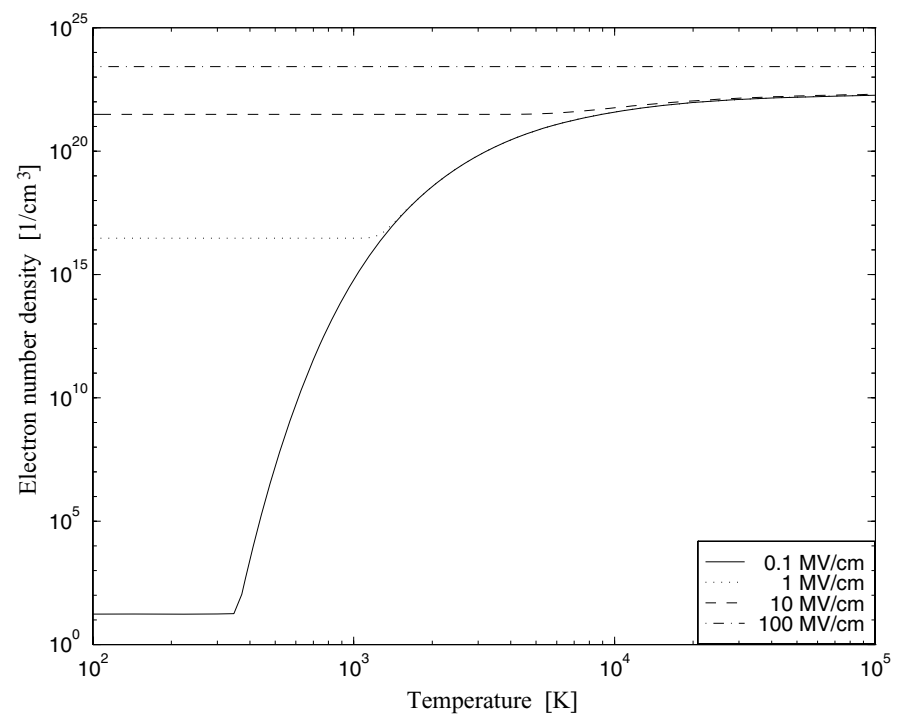

Fig. 2. Electron number density vs. temperature for four values of laser E field 


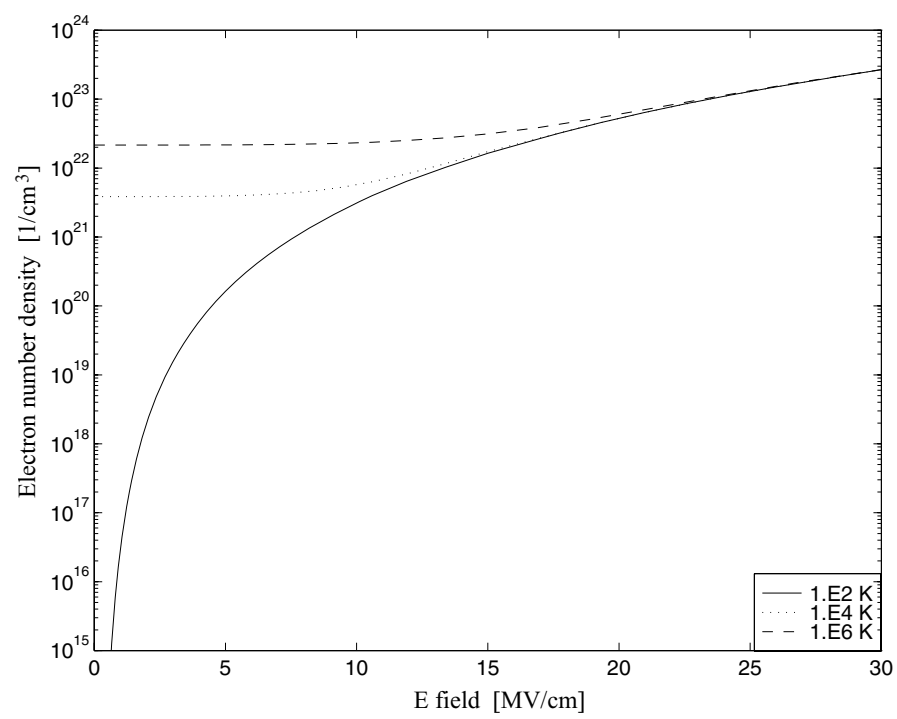

Fig. 3. Electron number density vs. laser E field for three temperatures

these equation for the electron number density, $n(E, T)$ results in the following equation,

$$
n(E, T)=\frac{1}{2 \sigma(E) v_{d r i f t}}\left(\alpha(E) V_{d r i f t}+\sqrt{\left(\alpha(E) V_{d r i f t}\right)^{2}+\left[2 \sigma(E) V_{d r i f t} n(T)\right]^{2}}\right)
$$

where $n(T)$ is the thermal generation given by eq. 5 .

The electron number density, given by eq. 8 is shown in Figs. 2, and 3, In our simulation, the average charge number, $Z$, was obtained by dividing the electron number density by the atom number density which was calculated by dividing the mass density by the average atomic mass.

Figure 3 shows that thermal generation of electrons is dominant in the low electric field region, while in the low temperature region electrons are excited by the laser electric field. In the shockwave experiment reported in this dissertation, the highest electric field was $8 \mathrm{MV} / \mathrm{cm}$ which corresponds to the laser energy at the highest enhancement factor with an applied fluence of $12 \mathrm{~J} / \mathrm{cm}^{2}$. Temperature in this simulation was up to a few $10,000 K$. For this case, the maximum electron number density was about $3-510^{22} \mathrm{~cm}^{-3}$.

\section{Conclusion}

To investigate the laser power absorption via electron heating, we form a physical model of laser energy absorption of glass through electron and derive the power 
transfer rate equation and the electron number density equation for a steady state as a function of temperature and electric field.

Numerical simulations show that the electron number density is strongly depends on the electric field strength and temperature at low temperature $(100 \mathrm{~K})$ and low electric filed strength $(0.1 \mathrm{MV} / \mathrm{cm})$. At high temperature and electric field regions, the dependency of the electron number density on electric field and temperature decreases. Further experimental and theoretical analysis is required for the breakdown conditions of glass through laser energy absorption.

\section{References}

1. E. Yablonovitch and N. Bloembergen, "Avalanche ionization and the limiting diameter of filaments induced by light pulses in transparent media," Phys. Rev. Lett., vol. 29, pp.907-910, 1972

2. L.H. Holway, Jr. and D.W. Fradin, "Electron avalanche breakdown by laser radiation in insulating crystals," Journal of Applied Physics, vol. 46, pp.279-291, 1975

3. S.C. Jones, P.Braunlich, R.T. Casper, X.A. Shen and P. Kelly, "Recent progress on laser-induced modifications and intrinsic bulk damage of wide-gap optical materials," Opt. Eng., vol. 28, pp.1039-1068, 1989

4. A. Epifanov, "Avalanche ionization induced in solid transparent dielectrics by strong laser pulses," Sov. Phys.-JETP, vol. 40, pp.897-9028, 1975

5. B. Gorshkov, A. Epifanov and A. Manenkov, "Avalanche ionization produced in solids by large radiation quanta and relative role of multiphoton ionization in laserinduced breakdown," Sov. Phys.-JETP, vol. 49, pp.309-315, 1979

6. D. Arnold and E. Cartier, "Theory of laser-induced free-electron heating and impact ionization in wide-band-gap solids," Physical review B, vol. 46, pp.15102-15115, 1992

7. D.A. Buchanan, M.V. Fischetti and D.J. DiMaria, "Coulombic and neutral trapping centers in silicon dioxide," Physcal Review B, vol. 43, pp.1471-1456, 1991

8. D. Du, X. Liu, G. Korn, J. Squier and G. Mourou, "Laser-induced breakdown by impact ionization in $\mathrm{SiO}_{2}$ with pulse widths from 7 ns to 150 fs," Appl. Phys. Lett., vol. 64, pp.3071-3073, 1994 